\title{
Designing Smart Home Interfaces for the Elderly
}

\author{
Zoraida Callejas, Ramón López-Cózar \\ Dep. Languages and Computer Systems \\ University of Granada (Spain) \\ \{zoraida,rlopezc\}@ugr.es
}

\begin{abstract}
In this paper we highlight the importance of tailoring the design of dialogue systems to the targeted user group. We propose a human-centered design cycle and report the results from a survey conducted among the intended users of a smart home for the elderly.
\end{abstract}

\section{Introduction}

The use of ubiquitous computing at home allows the automation of routine activities, removing physical barriers and adding benefits provided by mechanical and electrical technologies (Aghajan et al, 2009). This is very useful for the elderly, as the living in a smart home can ease their everyday activities and in some cases might be the only way of enjoying autonomy without risks.

To ease the interaction for these users, we are intending to set the basis for the design and development of a ubiquitous multimodal dialogue system to assist them in their daily home activities. Communication with computers using natural language eases the interaction notably and is a key issue in the efforts towards the so-called e-integration and eaccessibility initiatives. By means of these new technologies everybody will be able to interact with smart homes, especially people who have problems using these new technologies due to ignorance, disregard or disabilities (López-Cózar and Callejas, 2010).

\section{Our proposal to design for usability}

One key aspect for the development of interfaces is putting the emphasis on usability. Usability clearly differs from other system quality measures such as maintainability, reliability or portability in that the user point of view plays a determinant role in it. As a result, the objective of the interactive development cycle must not be only to obtain fully functional systems, but must also be concerned with the development of systems which adapt to the user needs, expectations and goals.

Thus, classic machine-centred approach, in which the system design and implementation are carried out following the technological aspects and functional user requirements, is no longer suitable. It becomes necessary to incorporate issues concerned with user capabilities, capacities, context and preferences which constitute the basis of humancentred design.

Several studies (Shafto and Brown, 2002) have shown that the life-cycle cost of complex systems is affected in approximately $70 \%$ by the decisions made in the early design stages. Thus, to mitigate life-cycle costs and risks it is very important to create user performance models that let the system designers quantify the real operation scenarios at the early design stages. 
Our proposal considers three key features of a system: interaction management, user modelling and multimodal interface. On the one hand, the interaction management establishes a relationship between system and context. Thus, e.g. to interpret a user gesture the system must have information about the dialogue and the social and situational contexts. On the other hand, the user models establish a relationship between users and their context. Thus, if the user is an elderly person, it is important to know e.g. whether s/he leaves alone and his/her disabilities. Finally, the multimodal interface provides a way for system-user communication.

We propose to carry out the tasks shown in Figure 1, which can be described as follows. In the first step, the system designers arrange a preliminary set of requirements based on their expertise on multimodal dialogue systems. In other words, they specify necessary and desirable functionalities and properties for the system. The second step is to refine these requirements taking into account the final users' points of view about the proposed functionality and characteristics, including also their new ideas and suggestions. The refined set of requirements allows in a third stage the creation of a good system specification. The fourth step is the construction of a first design of the system that complies with the specification. Finally, the fifth step uses the so-called Wizard of Oz (WOZ) technique (Dow et al, 2005) to find the usage context of the preliminary design, which is incorporated into posterior designs in an iterative way.

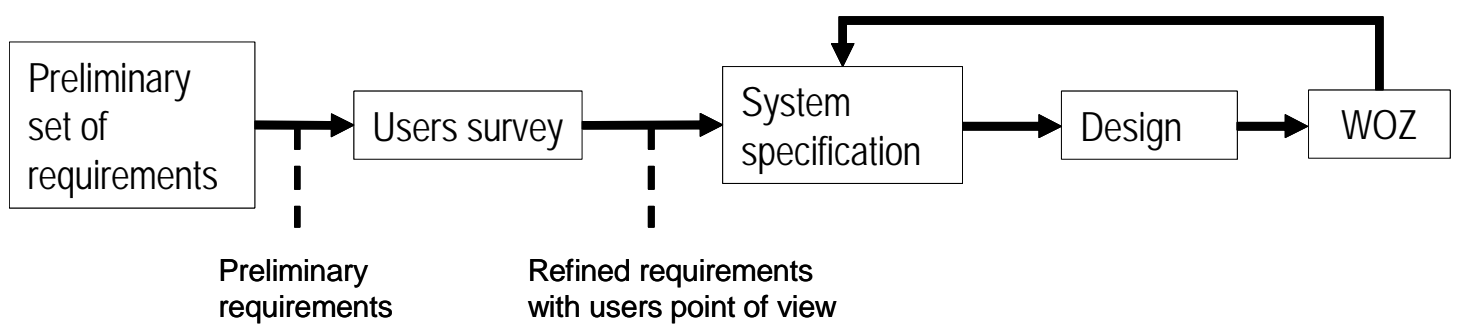

Figure 1. Tasks to implement the proposed approach to system design.

\section{A case study: design of an interface of a smart home for the elderly}

In the preliminary requirement analysis of our system we established the functional requirements to include the multimodal control of home appliances such as lamps, ovens, windows and heating. We also considered the system should include some entertainment functionality, in our case music and TV remote control and programming. We also found interesting that our multimodal system included a directory for helping the elderly users to make telephone calls. This way they could utter the name of the person they wish to call without remembering his/her telephone number.

Once we had restricted the problem, we reduced the possible solutions by establishing a set of non-functional requirements. Therefore, a suitable system that accomplished all the functional restrictions should also have the following characteristics: non intrusive, i.e. it would not interrupt users in their activities unless necessary, proactive, i.e. it would decide when to help users autonomously considering the environment's current status, adaptive, i.e. it would take into account the environment conditions in terms of noise and lighting to decide the most reliable interaction modalities. The system would also allow external communication, e.g., it could make emergency phone calls, and would accept incoming 
user calls to operate several devices (e.g. switching the heating on before the user arrives home). Finally, to enhance the user experience, it should be customizable, friendly and easy to use.

\section{Users survey}

To refine the preliminary requirement as shown in Figure 1, we carried a user survey among 200 men and women with ages ranging 50-80 who lived in towns of different sizes. Most interviewed were aged 60-70,58\% women and $42 \%$ men. The survey includes a representative sample of different segments of age, studies, economic situation and residence place, to obtain results that could be generalized for all the intended users of the system.

The survey was created following several style rules. The first was the usage of an easy language so that the questions it contained could be perfectly understood by every polled, potential user. Thus, we avoided foreign or very specialized words. As the survey was directed to elderly, we avoided asking about something that had been explained long ago, and employed very concise questions. Besides, questions were ordered from general to specific, clearly divided in different thematic areas. Due to the difficulty for the elderly to understand concepts related to new technologies, we explained every idea and illustrated it with a drawing representing a person interacting with the system.

We carried out the survey in two ways: one was by giving a printed copy of the survey to the polled, while the other was interviewing them orally. For the former we used large fonts for the text so that it was easy to read for them. For the latter, an interviewer read the form and took note of the polled answers and opinions.

Firstly, a section introduced the system we are planning to develop and explained why the user collaboration was very important for the design. Secondly, there was a section with questions related to personal details: age, gender, residence place, studies, labour situation, among others.

A third section contained questions about the utility of the different system functionalities were enclosed in seven categories: illumination, temperature, windows and blinds, music, television, kitchen and contact. Illumination referred to the ability of the system to control illumination via multimodal commands (e.g. switch the lights on and off), temperature referred to remote control of the house temperature, windows and blinds referred to remotely opening/closing them without the user having to make any effort. Music and TV were the system functions that enable the user to control and program the radio and TV sets (e.g. choose TV channels or radio stations). In the kitchen category we enclosed the control of the main home appliance such as ovens, washing machines and fridges. Finally, in the contact category we include the system's ability to act as a telephone agenda. For each of these categories, the survey started with a brief paragraph describing the system function followed by a drawing which explained the concept graphically. Then the polled people were asked if they found this function useful and how frequently they would use it. They were also asked to justify negative answers. Furthermore, a blank space allowed them to indicate other possible functionalities associated with the category.

The last part of the survey considered system characteristics rather than functionalities. The polled were asked to indicate if they found useful: i) the system's exterior access (e.g. to order switching the heating on before s/he arrives home), ii) proactiveness (the system 
ability to carry out tasks without being asked to, e.g. to remind things to the user by its own initiative), iii) human appearance (using an animated agent to generate a visual system output), iv) ease of use, v) customizability, vi) user location (system ability to know where the user is at every moment, e.g. to decide if s/he forgot to switch off a light) and vii) recovery from interaction errors. Finally the polled were asked to include comments or suggestions about the system functionality.

The survey results are shown in Figure 2 and 3, which illustrate the perceived usefulness of the main systems functionalities and their intended frequency of use respectively.

The figures show the results are very encouraging, as in all the cases the polled people found the functionalities useful, although their usage varies (e.g. music control was found useful but it would not be used very frequently). The telephone agenda was the most useful utility in the potential users' opinion, even for who indicated not suffering from memory problems. A reason for that might be that this kind of application is better known, so the polled distinguished with more confidence that it was really useful for them.

It was specially unexpected for us the acceptation of the windows and blinds function by the polled people, which was explained to them as the ability of the system to open and close windows and blinds automatically by means of voice commands. We think the reason for this result is that it is a very arduous task for the elderly. This is especially true for those who suffer from motor disabilities (124 out of 200 polled). Another very well accepted function is the TV control using spoken commands. We think the reason is that watching TV is the most widespread activity between the elderly (more than $90 \%$ of the Spanish older than 65 watch TV every day).

However, the ability of the system to control kitchen appliances (e.g. ovens) was not very well accepted as most people answered they would never use this function. We think the reason is that most of the elderly do not make the home duties alone (as they indicated in the first part of the survey), either because they share them with other family members, or because somebody does that work for them. We found that $50 \%$ of people who make all duties (usually women) considered this function really useful, while $69.4 \%$ of the polled who do not do the activities at home said they did not find it useful and would never use the system for those tasks.

We also found that, in general, all the system characteristics (non functional requirements) were broadly accepted: access from the house exterior (52\%), reminder of things to users $(91 \%)$, user location within the house (59\%), personalization (58\%), emergency calls (84\%). These results show the most broadly accepted system characteristic is its proactiveness. On the contrary, the animated agent was not well accepted (54\% of acceptance, $37 \%$ non acceptance) as many of the interviewed people remarked they preferred not to have a human image of the system when they interact with it. Furthermore, 95\% of interviewed said they would consider the system easy to use because of its multimodal interface.

The potential users were also asked what they would do if the system misunderstands them. Surprisingly, most said they would repeat the same thing until it finally understood. The top second answer was to shut the system down. This fact clearly shows the difficulties in developing a multimodal dialogue system for the elderly, as they would not trust the system if it fails. In fact, $5 \%$ of the interviewed would never use the system again in case of error. This result contrasts with the answers to the question about which aspect of the system they 
considered most important: well functioning, system kindness when it speaks or ease of use. Most said to prefer kindness and ease of use before correct functioning.

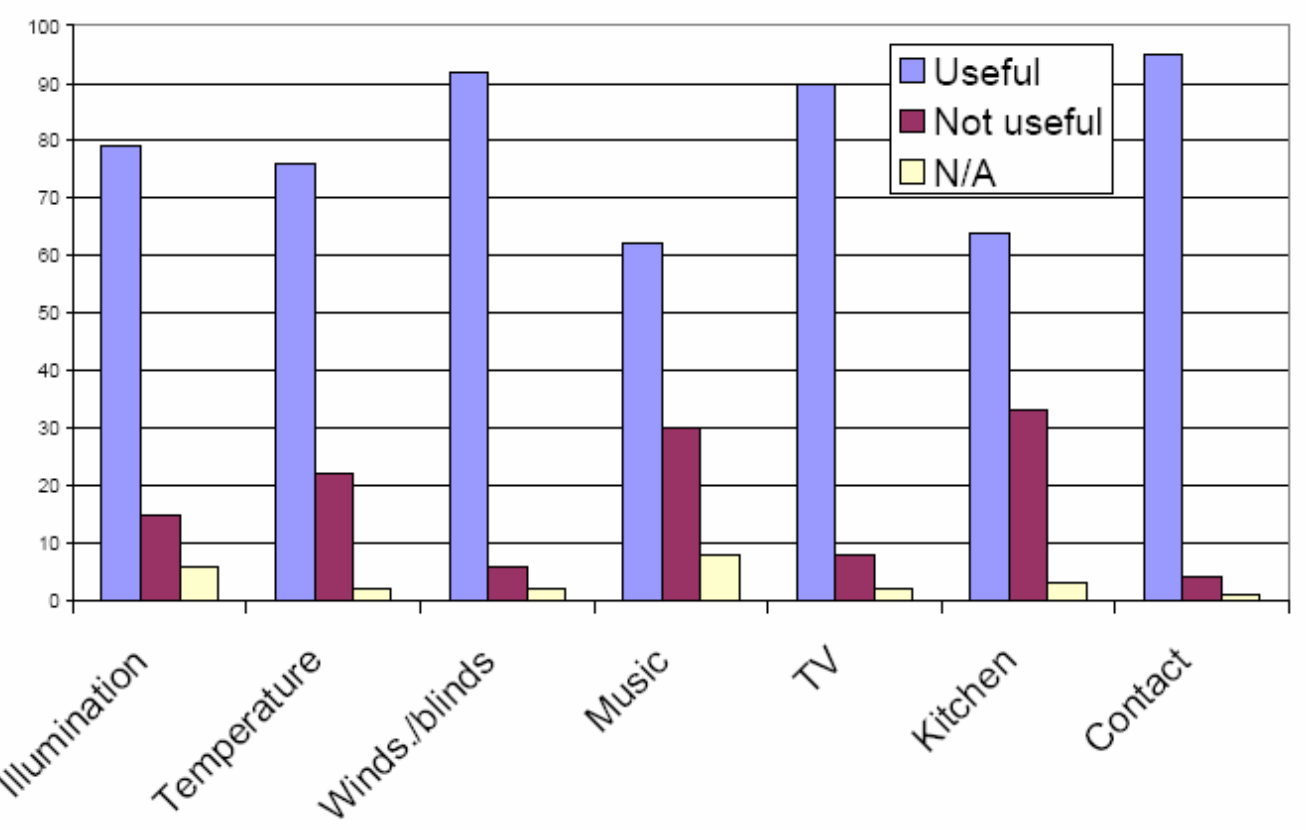

Figure 2. Perceived usefulness of the func tionalities.

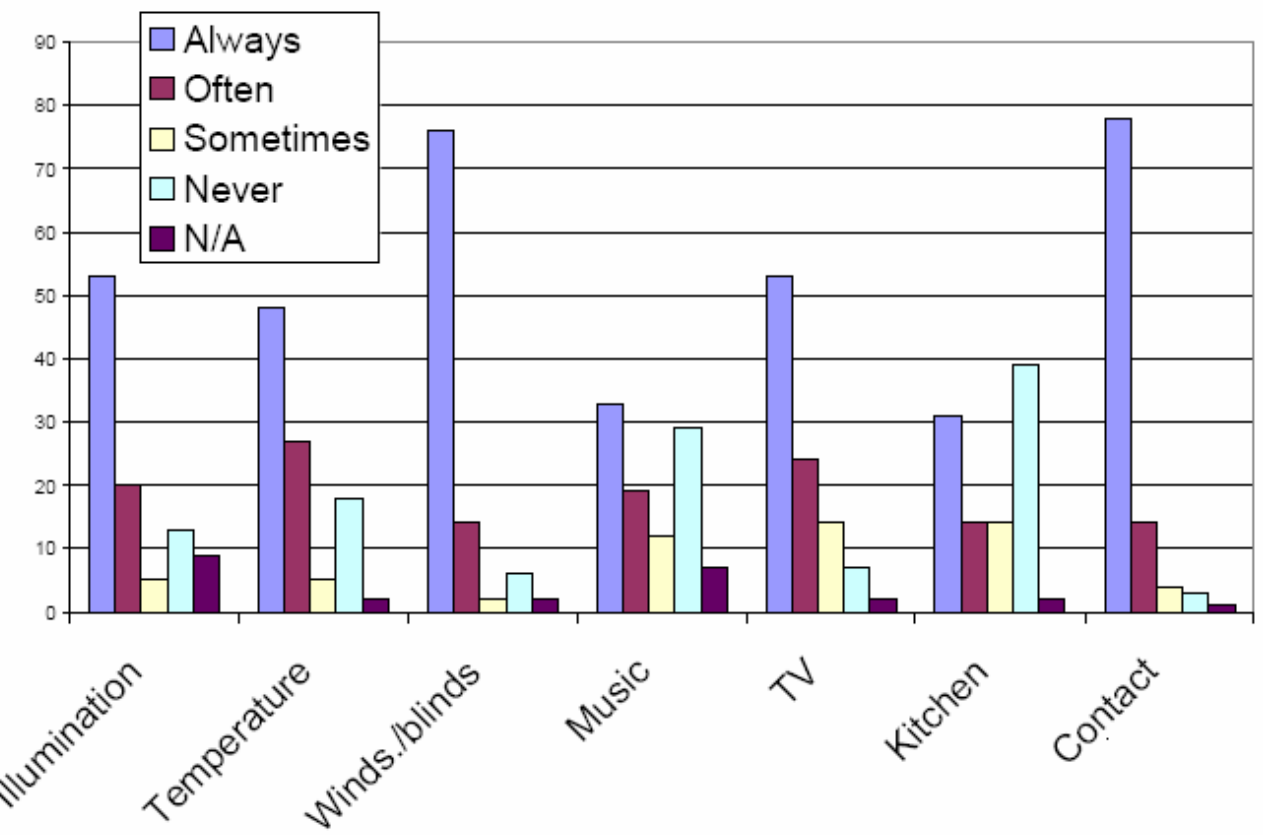

Figure 3. Intended frequency of use of system func tionalities. 
Finally, when the interviewed were asked to suggest new functionalities, it was surprising to discover their great imagination and how, in despite of their age, they were so open minded to discover totally unexpected pros and cons of the system. For example, related to the illumination control, some suggested that the system could activate an antiburglaring mechanism when there is nobody home. For example, turning on the lights could make believe that there is someone inside. Others pointed out that the system could evaluate the status of the house and its devices and warn the user if something is not working properly and find automatically somebody to fix the problem (e.g. phone an electrician or plumber). Another feature proposed by some polled is the ability to recognize face and voice not only for detecting the owner but also for recognizing his/her relatives. This way when somebody knocks at the door, the elderly would not have to move if it is not a family member.

When the elderly were interviewed in groups, some of them were able to find solutions to others' fears about this new technology. For example, as said before many of them did not want a human appearance for the system (e.g. an animated agent displayed on the TV screen) because they found it frightening, even when they were told that it could be a cartoon. Someone asked if they would be able to choose the agent face, and when we answered this could be possible, the subject said he would not be afraid if it had the face of his sons or grandsons. The others agreed with him.

Among the disadvantages of the system the youngest indicated that letting it do the work for them could led to loosing their skills sooner due to the lack of use. For example, they said they would loose memory if the system reminds them of all. We think studying this aspect could be a very interesting future work guideline once the system is working.

\section{Conclusions}

This paper has presented a new proposal to system design based on three main features: interaction management, user modelling and multimodal interface. This proposal is the result of merging previous strategies towards system specification and design. A very important factor of the new approach is taking the final users' needs and preferences into account right from the initial design states.

As a case of study, the approach has been partially applied to the design of a new multimodal dialogue system to assist elderly people is their daily home activities. The paper has shown how the functionalities and characteristics which a priori seemed to be more useful were not really considered as such by the interviewed potential users (e.g. employing animated agents to enhance the user experience). Moreover, it has shown that the potential users can point out new system features that the designers did not think of, such as automatic recognition of people knocking at the door.

\section{Acknowledgments}

This research has been funded by the project HADA TIN2007-64718 of the Spanish Ministry for Education and Science. 


\section{References}

1. H. Aghajan, J.C. Augusto, R. López-Cózar, Human-Centric Interfaces for Ambient Intelligence, Elsevier, 2009.

2. S. Dow, B. Maclntyre, J. Lee, C. Oezbek, J.D. Bolter, M. Gandy, Wizard of Öz Support throughout an Iterative Design Process, IEEE Pervasive Computing, November 2005.

3. R. López-Cózar, Z. Callejas, Multimodal Dialogue for Ambient Intelligence and Smart Environments. Handbook of Ambient Intelligence and Smart Environments, Springer, 2010.

4. M. Shafto, B. Brown, Human Centered Systems, Proc. AAAI 02, Edmonton (Canada), 2002.

\section{About the Authors}

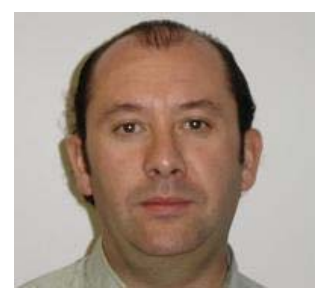

Ramon López-Cózar is Professor at the Faculty of Computer Science and Telecommunications of the University of Granada (Spain). His main research interests in the last 15 years include spoken and multimodal dialogue systems as well as Ambient Intelligence. He has coordinated several research projects, has published a number of journal and conference papers, and has been invited speaker at several scientific events addressing these topics. In 2005 he published the book "Spoken, Multilingual and Multimodal Dialogue Systems: Development and Assessment" (Wiley) and recently has co-edited the book "HumanCentric Interfaces for Ambient Intelligence" (Elsevier Academic Press, 2010). He is a member of ISCA (International Speech Communication Association), FoLLI (Association for Logic, Language and Information), AIPO (Spanish Society on Human-Computer Interaction) and SEPLN (Spanish Society on Natural Language Processing).

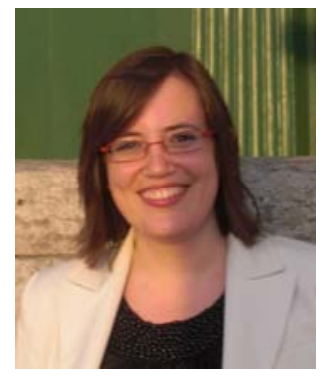

Zoraida Callejas is Assistant Professor in the Department of Languages and Computer Systems at the Technical School of Computer Science and Telecommunications of the University of Granada (Spain). She completed a PhD in Computer Science at University of Granada in 2008 and has been a visiting researcher in University of Ulster (Belfast, UK), Technical University of Liberec (Liberec, Czech Republic), University of Trento (Trento, Italy) and Technical University of Berlin (Berlin, Germany). Her research activities have been mostly related to speech technologies and in particular to the investigation of dialogue systems. Her results have been published in several international journals and conferences. She has participated in numerous research projects, and is a member of several research associations focused on speech processing and human-computer interaction. 